\title{
Isoelectric Focusing
}

National Cancer Institute

\section{Source}

National Cancer Institute. Isoelectric Focusing. NCI Thesaurus. Code C16758.

A type of gel electrophoresis in which charged molecules travel through a pH gradient.

The molecules will come to stop at the point where the net charge on the molecule is equal to that of the surrounding gel. 